\title{
USE OF AN OPEN-SOURCE SOFTWARE TO EXAMINE LOW SKELETAL MUSCLE MASS IN PENILE CANCER PATIENTS: A CROSS-SECTIONAL STUDY
}

\author{
C. Ibilibor, H. Wang, D. Kaushik, R. Rodriguez
}

\begin{abstract}
Purpose: Low skeletal muscle mass determined radiographically has emerged as an important prognostic marker in penile cancer patients but may be unrecognized in obese patients with a high comorbid disease burden. Moreover, publicly available software for image segmentation are limited. Thus, we describe the prevalence of radiographically low skeletal muscle mass in an obese penile cancer cohort, using an open-source software and examine its association with comorbid disease burden. Methods: This is a cross-sectional study, utilizing retrospective data from patients diagnosed with penile squamous cell carcinoma between October 2009 and December 2019. Available digital files of perioperative computerized tomography were analyzed, using CoreSlicer, anopen-sourceimagesegmentationsoftware. The correlationbetween radiographically low skeletalmusclemass, defined asa skeletal muscle index (SMI) less than $55 \mathrm{~cm}^{2} / \mathrm{m}^{2}$ and a Charlson Comorbidity Index (CCI) greater than 4 was examined, using logistic and linear regression. Results: Forty two of 59 patients had available digital files. Median SMI and body mass index (BMI) were $54.6 \mathrm{~cm}^{2} / \mathrm{m}^{2}$ and $30.2 \mathrm{~kg} / \mathrm{m}^{2}$ respectively for the entire cohort. Of included patients, $54 \%$ had radiographically low skeletal muscle mass and a median BMI of $28.9 \mathrm{~kg} / \mathrm{m}^{2}$. Radiographically low skeletal muscle mass was associated with a CCI greater than 4 on univariable and multivariable logistic regression with odds ratios of 4.85 ( $\mathrm{p}=0.041$ ) and $7.32(\mathrm{p}=0.033)$, respectively. When CCI was treated as a continuous variable on linear regression, the association between radiographically low skeletal muscle mass and CCI was positive, but not statistically significant with an estimated effect of $1.29(\mathrm{p}=0.1)$ and 1.27 ( $\mathrm{p}=0.152)$ on univariable and multivariable analysis, respectively. Conclusion: Our data demonstrate that low skeletal muscle mass can be readily assessed with CoreSlicer and is associated with a CCI greater than 4 in obese penile cancer patients.
\end{abstract}

Key words: Low skeletal muscle mass, obesity, penile cancer, computer software, charlson comorbidity index.

\section{Introduction}

Growing examination of the association between body composition and perioperative risk stratification has led to the identification of low skeletal muscle mass $(\mathrm{SMM})$ as an important diagnostic and prognostic marker in patients undergoing major surgery (1). While many different metrics have been put forth to define low SMM as a syndrome of low skeletal muscle quality and quantity, low SMM determined radiographically is marked principally by a low skeletal muscle index (SMI) where SMI is differentiated from SMM by being skeletal muscle surface area at the $3^{\text {rd }}$ lumbar normalized to height $(2,3)$. Within the oncologic literature, radiographically low SMM has been associated with increased rates of post-operative complications, cancer-

Department of Urology, University of Texas Health Science Center at San Antonio, San Antonio, TX, USA

Corresponding Author: Christine Ibilibor, M.D., M.Sc, Department of Urology, University of Texas Health Science Center at San Antonio, 7703 Floyd Curl Drive San Antonio, TX 78229, USA, Phone: 210567 5676, Fax: 210-567-6868, ibilibor@uthscsa.edu, ORCID: 0000-0002-3432-7883

Received May 4, 2021

Accepted for publication June 13, 2021 specific and all-cause mortality in multiple disease sites, including bladder, gastrointestinal and endometrial cancers (4-7). These poorer oncologic outcomes found in patients with low SMM have been reported to be, at least in part, due to the association between low SMM and higher chronic comorbid disease $(4,8)$. Increased usage of body composition as a corollary to clinical outcomes in different oncologic sites has led to an increasing number of image analysis platforms available for analytic morphomic. However, software commonly used for image segmentation such as Slice-O-Matic and OsiriX are proprietary and not readily accessible (9). Within the realm of urologic oncology, penile cancer patients have been reported as having the third highest median number of chronic comorbid conditions behind bladder and kidney cancer patients (10). Thus, penile cancer patients represent a population of men with a high comorbid disease burden by virtue of the modifiable patient related factors and such as cigarette smoking and obesity, where the latter is commonly associated with a sedentary lifestyle, that increase the risk of developing of penile cancer (11-13). However, due to the rarity of penile cancer there have been limited studies evaluating 
the association between low SMM, comorbid disease burden, oncologic and surgical outcomes in this patient population (14-16). We believe the rarity of this cancer type and the given high comorbid disease burden of these men warrants further investigation into the prevalence of low SMM among these patients and its correlation with indices of comorbidity. Within this context, our objective is to investigate the relationship between the presence of radiographically defined low SMM and comorbid disease burden in a penile cancer patient cohort, using an open-source web-based image segmentation software to validate its utility in a clinical population.

\section{Materials \& Methods}

\section{Patient Population}

This is a cross-sectional study, utilizing retrospective data. Thus, after institutional review board approval was obtained (HSC20190555H), patients diagnosed with penile squamous cell carcinoma (PSqCC) between October 2009 and December 2019 were consecutively identified within the University of Texas Health Science Center at San Antonio (UTHSCSA) health system. Among the identified PSqCC patients, only those who had undergone standard of care resection including but not limited to partial penectomy, total penectomy, and ILND were further identified using the following corresponding current procedural terminology (CPT) codes: 54120, 54125, 38765, 38531, and 38760. Approximately 59 patients treated for PSqCC were identified in the UTHSCSA system, of those, 42 had available digital files and were included for analysis. Clinicopathologic data were captured through a retrospective chart review with Charlson Comorbidity Index (CCI) scores calculated from abstracted patient data.

\section{Imaging Analysis}

The open-source web-based medical image segmentation platform, CoreSlicer, was used for image analysis. The digital files for perioperative computerized tomography (CT) scans performed within 90 days of the primary tumor resection or ILND were imported from the medical record and anonymized in digital imaging and communications in medicine (DICOM) format and subsequently uploaded into CoreSlicer. Only CT scan imaging obtained prior to ILND were used for patients who underwent this procedure. Within the CoreSlicer user interface, a single cross-sectional image obtained at the level of the $3^{\text {rd }}$ lumbar vertebrae was selected by an investigator $(\mathrm{CI})$ blinded to the patient's clinical data. Image segmentation at the $3^{\text {rd }}$ lumbar vertebrae was performed using a threshold brush that allows for the demarcation of regions of interest based on attenuation thresholds. Skeletal muscle attenuation thresholds ranged from -29 to +150 Hounsfield units while subcutaneous, visceral, and intramuscular adipose attenuation thresholds ranged from -190 to -30 Hounsfield units. SMI was calculated by normalizing the surface area of skeletal muscle in centimeters squared at the $3^{\text {rd }}$ lumbar vertebrae to height in meters squared. Fat mass index (FMI) was calculated by deriving total fat mass in kilograms from the sum total surface area of adipose in centimeters squared which included subcutaneous, visceral and intramuscular adipose, using the below equation and normalizing it to height in meters squared (17).

Total fat mass $(\mathrm{kg})=\left(\right.$ Total adipose area at the $3^{\text {rd }}$ lumbar in $\left.\mathrm{cm}^{2}\right) \times 0.042+11.2$

\section{Primary Predictor Variables}

The primary objective of this study is to determine whether there is an association between radiographically low SMM and a CCI greater than 4; an association between SMI and CCI has been reported previously (8). Low SMM, defined as an SMI less than $55 \mathrm{~cm}^{2} / \mathrm{m}^{2}$, served as our primary predictor. This cut-off was chosen due to its previously reported use in this patient population (15). Also, we treated low SMM as a categorical variable in our analysis due to its previous use in this patient population and our clinical practice (15). Similarly, obesity defined as an FMI greater than $10 \mathrm{~kg} / \mathrm{m}^{2}$, based on the NHANES 2009 criteria, or a body mass index (BMI) greater than 30 $\mathrm{kg} / \mathrm{m}^{2}$ served as secondary predictors of elevated CCI (18).

\section{Primary Outcome Variables}

The CCI for each patient was calculated by using abstracted patient data and served as the primary outcome variable because it has been previously demonstrated to be an important marker of comorbid disease burden $(19,20)$. The CCI is based on patient age, and the presence of 16 diseases with varied disease specific severity measures. One point is given for an age between 50-59, and one additional point for each decade above 50 up to 80 years. One point was given for history of myocardial infarction, congestive heart failure, peripheral vascular disease, cerebrovascular accident, dementia, chronic obstructive pulmonary disease, peptic ulcer disease, liver cirrhosis without portal hypertension (3 points for cirrhosis with portal hypertension, with or without a history of variceal bleeding), and history of uncomplicated diabetes mellitus ( 2 points for diabetes mellitus with end organ damage). Similarly, two points were given for presence of a localized tumor (6 points for metastatic disease), 2 points for leukemia and lymphoma while 6 points were given for the presence of acquired immunodeficiency syndrome. Patients with a CCI greater than 4 points were defined as having a high comorbid disease burden based on previous literature in the penile cancer population (15). 
Table 1

Baseline patient characteristics stratified by the presence of Low Skeletal Muscle Mass

\begin{tabular}{|c|c|c|c|}
\hline & $\begin{array}{c}\text { Low SMM } \\
\quad(n=23)\end{array}$ & $\begin{array}{c}\text { Normal SMM } \\
(n=19)\end{array}$ & $p$ value \\
\hline Median age, years (IQR) & $71(60-74)$ & $54(49-62)$ & $<0.001$ \\
\hline Median SMI, $\mathrm{cm}^{2} / \mathrm{m}^{2}$ (IQR) & $\begin{array}{c}47.9(43.6- \\
51.7)\end{array}$ & $\begin{array}{l}63.3(60.8- \\
68.4)\end{array}$ & $<0.001$ \\
\hline Median FMI, $\mathrm{kg} / \mathrm{m}^{2}$ (IQR) & $11.7(8.9-14.8)$ & $11.4(9.3-12.9)$ & 0.924 \\
\hline Median BMI, kg/m2 (IQR) & $28.9(24.5,34.0)$ & $31.3(28.5,33.1)$ & 0.323 \\
\hline Smoking History, n (\%) & & & 0.379 \\
\hline Non-smoker & $8(34.8)$ & $8(42.1)$ & \\
\hline Current Smoker & $4(17.4)$ & $5(26.3)$ & \\
\hline Former Smoker & $11(47.8)$ & $6(31.6)$ & \\
\hline Median CCI (IQR) & $6(5-9)$ & $5(3-9)$ & 0.066 \\
\hline CCI, n (\%) & & & 0.075 \\
\hline $2-4$ & $3(13)$ & $8(42.1)$ & \\
\hline$>4$ & $20(87)$ & $11(57.9)$ & \\
\hline Ethnicity, n (\%) & & & 0.228 \\
\hline Hispanic & $13(56.5)$ & $15(78.9)$ & \\
\hline Non-Hispanic & $10(43.5)$ & $4(21.1)$ & \\
\hline Race, n (\%) & & & 1.000 \\
\hline White & $22(95.5)$ & $19(100.0)$ & \\
\hline Non-white & $1(4.5)$ & $0(0)$ & \\
\hline Primary Tumor Stage, n (\%) & & & 0.077 \\
\hline Tis $-\mathrm{T} 1 \mathrm{~b}$ & $5(23.8)$ & $9(47.4)$ & \\
\hline $\mathrm{T} 2$ & $6(26.1)$ & $7(36.8)$ & \\
\hline $\mathrm{T} 3$ & $12(52.2)$ & $3(15.8)$ & \\
\hline Presence of LVI, n (\%) & & & 0.186 \\
\hline Yes & $9(39.1)$ & $3(15.8)$ & \\
\hline No & $14(61.9)$ & $16(84.2)$ & \\
\hline Tumor Differentiation, n (\%) & & & 0.840 \\
\hline Well & $6(26.1)$ & $6(31.6)$ & \\
\hline Moderate & $13(56.5)$ & $9(47.4)$ & \\
\hline Poor & $4(17.4)$ & $4(21.1)$ & \\
\hline ILN Management, n (\%) & & & 0.977 \\
\hline Surveillance & $8(34.8)$ & $7(36.8)$ & \\
\hline ILND & $12(52.2)$ & $10(52.6)$ & \\
\hline Radiation & $2(8.7)$ & $1(5.3)$ & \\
\hline Chemotherapy & $1(4.8)$ & $1(5.3)$ & \\
\hline Median follow-up, years (IQR) & $0.6(0.3-1.9)$ & $1.2(0.2-2.7)$ & 0.488 \\
\hline
\end{tabular}

BMI: body mass index, CCI: Charlson Comorbidity Index, FMI: fat mass index, ILN inguinal lymph node, ILND: inguinal lymph node dissection, IQR: interquartile range, LVI: lymphovascular invasion, SMI: skeletal muscle index, SMM: skeletal muscle mass

\section{Statistical Analysis}

Categorical variables such as low SMM, smoking status and ethnicity were expressed as percentages, while continuous variables such as SMI, and BMI were expressed as medians and interquartile ranges. Predictor variables, low SMM, smoking history, ethnicity, and obesity, were chosen a priori, and used to model our primary outcome variable. Univariable and multivariable linear regression models were used to model the effect of our predictors on CCI as a continuous variable while univariable and multivariable logistic regression models were used to model CCI greater than 4 as a categorical variable. Age was not used as a predictor variable nor included in our regression models because age is incorporated into CCI calculations; thus inclusion of age in any analyses modeling CCI would be over adjusted. All statistical analyses were performed in R Version 4.0.0 (Vienne, Austria). All tests were two-sided and a $p$-value less than 0.05 was the criterion for statistical significance.

Figure 1

Representative CoreSlicer images of four different body compositions

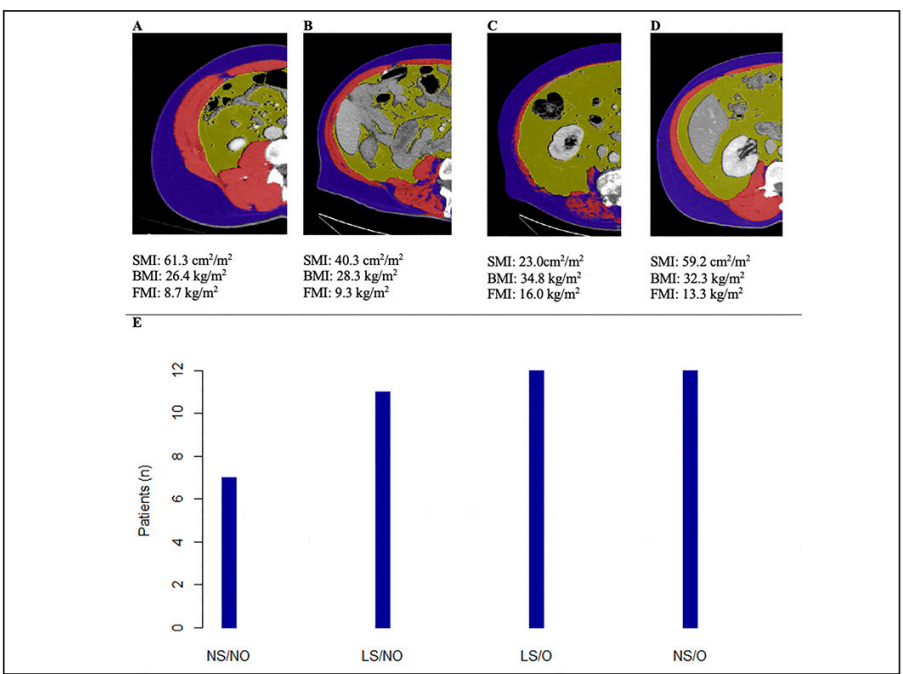

A) normal skeletal muscle mass non-obese, B) low skeletal muscle mass non-obese, C) low skeletal muscle mass obese, D) normal skeletal muscle obese, and with visible differences in the skeletal muscle (red), visceral adipose (yellow) and subcutaneous (blue) surface areas. E) bar graph demonstrating the number of patients in each category. BMI: body mass index, FMI: fat mass index, LS/NO: low skeletal muscle mass/non-obese, LS/O: low skeletal muscle mass / obese, NS/NO: normal skeletal muscle mass/non-obese, NS/O: normal skeletal muscle mass / obese, SMI: skeletal muscle index

\section{Results}

Cohort data. The clinicopathologic characteristics of the 42 included stratified by presence of radiographically low SMM are summarized in Table 1 . The 17 patients without available digital files had similar baseline demographics to those of the included patients (data not shown). Median SMI, BMI and FMI for the entire cohort were $54.62 \mathrm{~cm}^{2} / \mathrm{m}^{2}, 30.2 \mathrm{~kg} / \mathrm{m}^{2}$ and $11.49 \mathrm{~kg} / \mathrm{m}^{2}$ respectively and $54 \%$ of patients had radiographically low SMM. Low SMM and normal SMM patients were largely similar based on FMI, BMI, smoking history, ethnicity/race, and inguinal lymph node management with one patient within each group receiving chemotherapy for inguinal and distant metastases at presentation. However, low SMM patients were generally older with a slightly higher median CCI score that approached statistical significance and a slightly higher proportion of pathologic T3 disease 
Table 2

Odds ratios for likelihood of having a CCI greater than 4

\begin{tabular}{|c|c|c|c|c|}
\hline & Univariable & & Multivariable & \\
\hline Low SMM & $4.85(1.06-22.11)$ & 0.041 & $7.32(1.17-45.66)$ & 0.033 \\
\hline Smoking History, Non-smoker & ref & & ref & \\
\hline Current Smoker & $0.46(0.10-2.22)$ & 0.335 & $0.89(0.09-8.48)$ & 0.485 \\
\hline Hispanic & $1.58(0.43-5.86)$ & 0.492 & $2.82(0.44-17.93)$ & 0.273 \\
\hline Obesity (FMI >10 kg/m²) & $1.15(0.29-4.61)$ & 0.839 & $2.55(0.41-35.62)$ & 0.441 \\
\hline Obesity (BMI >40 kg/m²) & $0.71(0.20-2.58)$ & 0.608 & $0.53(0.06-4.57)$ & 0.560 \\
\hline
\end{tabular}

BMI: body mass index, CCI: Charlson Comorbidity Index, CI: confidence interval, FMI: fat mass index, OR: odds ratio, SMM: skeletal muscle mass

Table 3

Estimated effects of predictors on CCI

\begin{tabular}{|c|c|c|c|c|}
\hline & Univariable & & Multivariable & \\
\hline Low SMM & $1.29(0.77)$ & 0.100 & $1.27(0.87)$ & 0.152 \\
\hline Smoking History, Non-smoker & ref & & ref & \\
\hline Current Smoker & $-0.25(0.91)$ & 0.779 & $0.74(1.20)$ & 0.543 \\
\hline Hispanic & $0.32(0.73)$ & 0.667 & $0.07(0.92)$ & 0.934 \\
\hline FMI & $0.01(0.12)$ & 0.952 & $-0.04(0.23)$ & 0.857 \\
\hline BMI & $-0.02(0.05)$ & 0.737 & $0.05(0.11)$ & 0.686 \\
\hline
\end{tabular}

BMI: body mass index, CCI: Charlson Comorbidity Index, SE: standard error, SMI: skeletal muscle index, FMI: fat mass index

that similarly trended toward statistical significance. Patients were further subdivided into four body categories based on BMI, FMI and SMI to determine the proportion of obese patients who had radiographically low SMM. Within the cohort, $28.6 \%$ were low SMM obese, $28.6 \%$ were normal SMM obese, while $26.2 \%$ were low SMM non-obese, and $16.7 \%$ were normal SMM nonobese. Figure 1 illustrates the number of patients within each of the four body categories with their representative image after image segmentation in CoreSlicer.

\section{Inguinal lymph node dissection subgroup}

Twenty-two patients underwent ILND and their clinicopathologic data are shown in Supplemental Table 1. Of note, among the patients classified with radiographically low SMM, a larger proportion underwent only bilateral superficial inguinal lymph node dissection and did not undergo sartorius flap creation. Thus, there was higher percentage of 30-day ILNDrelated complications in the normal SMM group.

\section{The association between low SMM and CCI}

On univariable and multivariable logistic regression analysis, radiographically low SMM had a strong association with CCI greater than 4 while smoking history, Hispanic ethnicity, and obesity defined by FMI and BMI had no association with CCI greater than 4 (Table 2). When CCI was treated as a continuous variable, the estimated effect of low SMM on CCI demonstrated a non-statistically significant positive correlation on univariable and multivariable linear regression (Table 3).

\section{Discussion}

In the present cross-sectional study, we utilize a new web-based open-source image segmentation platform called CoreSlicer in a clinical population which to our knowledge has not be reported previously. This software has been shown to provide image analysis comparable to other proprietary software such as Slice$\mathrm{O}$-Matic and lends itself to higher ease of use through its 
wizard format, allowing it to be potentially integrated into a clinical work-flow. CoreSlicer positions itself as a platform for radiomics and the extraction of highquality data from clinical imaging (9). The clinical value of this open-source software is high given its accessibility; however, it is currently only intended for use in investigative research. Thus, further investigation of CoreSlicer's use in clinical populations such as the one presented here is warranted.

Here, we also report the prevalence of low SMM in an obese penile cancer population and a nearly 5-fold increased likelihood of high comorbid disease burden in men with low SMM alone and a greater than 7-hold increased likelihood when adjusted for other covariates. A negative linear correlation between CCI and SMI has between reported in a geriatric medical patient population with patients demonstrating a higher CCI and poorer performance status also exhibiting lower SMI values (8). Our study helps confirm this phenomenon in a highly comorbid cancer population.

Penile cancer patients represent a population of men with a high comorbid disease burden owing to the risk factors that are associated with penile cancer development such as cigarette smoking and obesity (11, 12). Thus, the impact of a sedentary lifestyle and overall inactivity on penile cancer risk through its contribution to obesity development, cannot be understated (13). Moreover, obesity represents a modifiable risk factor for penile cancer development (11). In the current study, most men were either obese with a low SMM or obese with a normal SMM, pointing to the prevalence of an elevated BMI in this population. It is currently unclear how lifestyle changes or a change in BMI effect penile cancer development, however further research in this area is warranted.

This study's limitations include its small sample size and retrospective design, leaving it susceptible to selection bias. However, the baseline demographic data for patients who did not have available digital files for analysis were similar to those in the studied cohort. Also, associations that approached statistical significance in our study may be accentuated in a larger sample size. In addition, our usage of CCI as a categorical variable can create an unbalanced population sample as those above and below our predefined threshold will differ inherently based on age.

\section{Conclusion}

In this study, we offer insight into the utilization of a publicly available software for body segmentation and the evaluation for the presence of low SMM in a surgical population with a characteristically high comorbid disease burden.
Conflict of interest: The authors have no conflict of interests to declare, financial or otherwise.

Ethical standard: The authors declare that the study procedures adhere to all ethical standards. Ethics approval for this study was obtained from The University of Texas Health Science Center at San Antonio Instituitional Review Board.

Acknowledgments: Christine Ibilibor has received research support through the University of Texas Health Science Center at San Antonio Cancer Research Training Program supported by the CPRIT Research Training Award (RTA; RP170345).

\section{References}

1. Friedman, J., Lussiez, A., Sullivan, J., Wang, S. \& Englesbe, M. Implications of sarcopenia in major surgery. Nutr Clin Pract 2015; 30, 175-179, doi: $10.1177 / 0884533615569888$.

2. Cruz-Jentoft, A. J. et al. Sarcopenia: revised European consensus on definition and diagnosis. Age Ageing 2019; 48, 16-31, doi:10.1093/ageing/afy169.

3. Dodds, R. \& Sayer, A. A. Sarcopenia and frailty: new challenges for clinical practice. Clin Med (Lond) 2016; 16, 455-458, doi:10.7861/ clinmedicine.16-5-455.

4. Kuroki, L. M. et al. Pre-operative assessment of muscle mass to predict surgical complications and prognosis in patients with endometrial cancer. Ann Surg Oncol 2015; 22, 972-979, doi:10.1245/s10434-014-4040-8.

5. Psutka, S. P. et al. Mortality after radical cystectomy: impact of obesity versus adiposity after adjusting for skeletal muscle wasting. J Urol 2015; 193, 15071513, doi:10.1016/j.juro.2014.11.088

6. Simonsen, C. et al. Sarcopenia and Postoperative Complication Risk in Gastrointestinal Surgical Oncology: A Meta-analysis. Ann Surg 2018; 268 58-69, doi:10.1097/SLA.0000000000002679.

7. Smith, A. B. et al. Sarcopenia as a predictor of complications and survival following radical cystectomy. J Urol 2014; 191, 1714-1720, doi:10.1016/j. juro.2013.12.047

8. Gong, G. et al. Correlation between the Charlson comorbidity index and skeletal muscle mass/physical performance in hospitalized older people potentially suffering from sarcopenia. BMC Geriatr 2019; 19, 367, doi:10.1186/ s12877-019-1395-5.

9. Mullie, L. \& Afilalo, J. CoreSlicer: a web toolkit for analytic morphomics. BMC Med Imaging 2019; 19, 15, doi:10.1186/s12880-019-0316-6.

10. Garg, T. et al. Burden of Multiple Chronic Conditions among Patients with Urological Cancer. J Urol 2018; 199, 543-550, doi:10.1016/j.juro.2017.08.005.

11. Barnes, K. T. et al. Obesity is associated with increased risk of invasive penile cancer. BMC Urol 2016; 16, 42, doi:10.1186/s12894-016-0161-7.

12. Daling, J. R. et al. Penile cancer: importance of circumcision, human papillomavirus and smoking in in situ and invasive disease. Int J Cancer 2005; 116, 606-616, doi:10.1002/ijc.21009.

13. Kushi, L. H. et al. American Cancer Society Guidelines on nutrition and physical activity for cancer prevention: reducing the risk of cancer with healthy food choices and physical activity. CA Cancer J Clin 2012; 62, 30-67, doi:10.3322/ caac. 20140 .

14. Christodoulidou, M. et al. Is sarcopenia a useful prognostic indicator in patients with squamous cell carcinoma of the penis? Journal of Clinical Oncology 2017; 35, 426-426, doi:10.1200/JCO.2017.35.6 suppl.426.

15. Sharma, P. et al. Sarcopenia as a predictor of complications in penile cancer patients undergoing inguinal lymph node dissection. World J Urol 2015; 33, 1585-1592, doi:10.1007/s00345-014-1471-6.

16. Takkamoto, D. et al. A low psoas muscle volume is associated with a poor prognosis in penile cancer. Oncotarget 2020; 11, 3526-3530, doi:10.18632/ oncotarget.27719.

17. Mourtzakis, M. et al. A practical and precise approach to quantification of body composition in cancer patients using computed tomography images acquired during routine care. Appl Physiol Nutr Metab 2008; 33, 997-1006, doi:10.1139/H08-075

18. Kelly, T. L., Wilson, K. E. \& Heymsfield, S. B. Dual energy X-Ray absorptiometry body composition reference values from NHANES. PLoS One 2009; 4, e7038, doi:10.1371/journal.pone.0007038.

19. Charlson, M. E., Pompei, P., Ales, K. L. \& MacKenzie, C. R. A new method of classifying prognostic comorbidity in longitudinal studies: development and validation. J Chronic Dis 1987; 40, 373-383, doi:10.1016/0021-9681(87)90171-8.

20. Quan, H. et al. Updating and validating the Charlson comorbidity index and score for risk adjustment in hospital discharge abstracts using data from 6 countries. Am J Epidemiol 2011; 173, 676-682, doi:10.1093/aje/kwq433. 